\title{
ProQuest
}

Databases selected: Research Library

\section{Uses and Effects of Mobile Computing Devices in K-8 Classrooms}

Karen Swan, Mark van 't Hooft, Annette Kratcoski, Darlene Unger. Journal of Research on Technology in Education. Eugene: Fall 2005. Vol. 38, Iss. 1; pg. 99, 14 pgs

\begin{abstract}
Summary)
This preliminary study employed mixed methodologies to explore students' use of mobile computing devices and its effects on their motivation to learn, engagement in learning activities, and support for learning processes. Data collected from students in four elementary and two seventh grade science classes in Northeast Ohio included usage logs, student work samples, student and teacher interviews, and classroom observations. Findings highlight the personalization of learning afforded by such devices both in terms of individuals and individual classroom cultures, as well as their usefulness in extending learning beyond the classroom. They also suggest that increased motivation due to mobile device use leads to increases in the quality and quantity of student work. [PUBLICATION ABSTRACT]
\end{abstract}

Full Text (5934 words)

Copyright International Society for Technology in Education Fall 2005

\begin{abstract}
[Headnote]
Abstract

This preliminary study employed mixed methodologies to explore students' use of mobile computing devices and its effects on their motivation to learn, engagement in learning activities, and support for learning processes. Data collected from students in four elementary and two seventh grade science classes in Northeast Ohio included usage logs, student work samples, student and teacher interviews, and classroom observations. Findings highlight the personalization of learning afforded by such devices both in terms of individuals and individual classroom cultures, as well as their usefulness in extending learning beyond the classroom. They also suggest that increased motivation due to mobile device use leads to increases in the quality and quantity of student work.

(Keywords: mobile computing, motivation, writing.)
\end{abstract}

\section{BACKGROUND}

More than a decade ago, Mark Weiser (1991) wrote that we live in a society in which technology is so pervasive that we do not notice it anymore when used for everyday tasks such as information retrieval, communication, and entertainment. Defining this environment as ubiquitous computing, he described it more as a state of mind, as "a new way of thinking about computers in the world ... [that] allows the computers themselves to vanish into the background ... [and] become indistinguishable from everyday life" (p. 94). As a result, the current generation of K12 students is growing up more technologically literate than children their age were a decade ago, with access to an increasing number of devices and services such as video game consoles, mobile gaming devices, cell phones, the Internet, and instant messaging. Interestingly enough, even though many students know and use these technologies as integral parts of their lives, they learned to do so mostly outside of school (National Educational Technology Plan, 2004), and teachers are struggling to integrate technology into their curriculum.

However, there are signs that the idea of ubiquitous computing is starting to get a foothold in $\mathrm{K}-12$ settings, as a vision of classrooms filled with many computing devices designed for differing purposes and to be used as needed in the same ways as pencils and paper and books are used now. This vision is accompanied by a need for systematic research to investigate its effect, which is especially important given the argument that technology can play a more significant role in education and everyday life if it becomes more human-centered and less visible. For learning, the implication is that the smaller and less disruptive the device, the more of a chance it stands of becoming a lifelong-learning tool for anyone, anywhere, anytime (Inkpen, 2001; Sharpies, 2002).

Given this theoretical framework and their relatively low cost, handheld computers are becoming an increasingly compelling choice of technology for K-12 classrooms because they enable a transition from the occasional, supplemental use of classroom computers and school computer labs to the frequent, integral use of portable computational devices (Soloway et al., 2001; Tinker \& Krajcik, 2001). Early evaluations indicate that teachers and 
students respond favorably to handheld devices, and suggest handheld computers have the potential to affect student learning positively across curricular topics and instructional activities. Teachers, for example, have indicated that students are more motivated, spend more time using technology, collaborate and communicate more, and benefit from having a portable and readily accessible tool (Vahey \& Crawford, 2002). Students, in turn, have found handhelds easy to use, fun, and a useful tool for learning (van 't Hooft, Diaz, \& Swan, 2004).

Perhaps more important, some researchers argue that classrooms with handheld computers differ fundamentally from more traditional desktop computing environments in that users interacting with handheld computers can also interact with each other and other computing devices at the same time (Cole \& Stanton, 2003; Danesh, Inkpen, Lau, Shu, \& Booth, 2001; Mandryk, Inkpen, Bilezkjian, Klemmer, \& Landay, 2001; Roth, 2002). Handheld computers thus have the potential to support both personalized and collaborative learning. Roschelle and Pea (2002), for example, highlight three ways handheld devices have been used to increase learning collaborativelyclassroom response systems, participatory simulations, and collaborative data gathering-and suggest there are many more such uses (Danesh et al., 2001; Mandryk et al., 2001; Roschelle, 2003).

Finally, because of their small size, handheld computing devices no longer constrain users in the way desktop computers or even laptops do. As such, handheld computers support learning outside the classroom, twenty-four hours a day, seven days a week. They thus have the potential to support lifelong-learning anywhere, anytime (Bannasch, 1999; Inkpen, 2001; Sharpies, 2002; Soloway et al, 2001; Staudt \& Hsi, 1999; Tinker, 1997).

However, the limited size of most handhelds may be a disadvantage as well as an advantage (van 't Hooft et al., 2004). Screen size is an issue for some, but text input on handheld computers is a more pressing one, especially at the elementary level. Unless students attach an external keyboard, which costs more money, takes up space, and affects mobility, text input is limited to the onscreen keyboard or text recognition software (Vahey \& Crawford, 2002). Primary teachers, in particular, believe that text recognition software can confuse students who are learning to write (van 't Hooft et al, 2004). In contrast, mobile computing devices such as the ones used in this study 1 may be the best of both worlds. On one hand, they feature a handheld-specific operating system, integrated wireless capabilities, and a full-size keyboard, and therefore function like handheld computers without the text input issues. On the other hand, they are relatively small and lightweight, and are cheaper and easier to use than fullblown laptop computers.

Research on the effects of mobile computers on teaching and learning is still relatively scarce (van t' Hooft et al., 2004). This preliminary study was designed to begin exploring the use of mobile computing devices and its effects on student learning. The following questions were addressed:

1.How do students use mobile computing devices?

2.Does the use of mobile computing devices affect students' motivation to learn and engagement in learning?

3.Does students' use of mobile computing devices support learning processes?

\section{METHODOLOGY}

\section{Subjects and Settings}

Data were collected from subjects at two sites during the 2003-2004 school year. The first site was a technologyrich laboratory classroom at a state university in northeast Ohio where local teachers (who are nominated by their administrators and subjected to a selection process) bring their classes to complete regular units of study in a ubiquitous computing environment. Classes spend half a day every day for six weeks in the classroom, with access to desktops, wireless laptops, and handheld computers (1:1), a document camera, a presentation system, scanners, printers, digital cameras, teleconferencing equipment, video and audio recorders, VCRs, video editing equipment, CD and DVD burners, digital microscopes, scientific probes, wireless writing pads, and a wide variety of software to support teaching and learning.

Classes and subjects involved at the first site included one sixth grade class $(n=28)$, two fourth grade classes $(n=$ $41)$, and one third grade class $(n=16)$. The sixth grade class's work centered on a biography project; one of the fourth grade classes studied plants and the environment; the other fourth grade and third grade classes, both from the same district, worked on identical projects organized around a study of flight. All students were given mobile computing devices to use and take home for the six-week period their classes spent in the laboratory classroom. In this part of the study, we were especially interested in the ways in which the use of mobile computing devices might support student development of conceptual understanding. Thus, representative students (high achieving, middle, low, special needs) in each class were closely followed. Representative students were identified by their teachers.

The second site was a suburban middle school in northeast Ohio whose student population of approximately 380 is drawn from two elementary schools, one attended by children from upper middle class, white-collar families and the 
other attended by middle class, blue-collar families. The school's students are primarily (about 98\%) Caucasian, and at the time of the study, about $20 \%$ of students qualified for free or reduced lunch. Students $(n=50)$ in two (out of five) seventh grade science classes, all taught by the same teacher, were given mobile computing devices to use in science and to take with them for a little more than half the school year. In this part of the study, we focused on the support mobile computing devices might provide in science learning, both in terms of motivation as well as conceptual understanding.

\section{Data Sources and Analyses}

Data collected from all six classes included lesson plans, usage data, work samples, student and teacher interviews, and classroom observations, some of which were videotaped. To answer the first research question, usage data was collected from all students for whom it was available using Rubberneck, a hidden software tool that collects usage data from individual devices. Local transfer of mobile device data to desktop computers sends this data to an off-site server that is accessible through the Internet. Usage data for each student was categorized by application. Because time periods for which usage data was collected varied among students, the aggregated data was then divided by total time of use to yield average use per week for each student. Averages were compared by classes and gender within classes to provide a detailed quantitative portrait of how students used their mobile computing devices both in and outside of class.

Usage data were triangulated with responses from student interviews. Students in all classes were interviewed concerning their use of mobile computing during the final weeks of their classroom experiences. Although the question and interview formats varied somewhat according to age and ability level and between instructional contexts (i.e., laboratory classroom interviews were conducted with individual students as they were working on projects, middle school interviews were conducted with selected students during class time, but separately from class work), all interviews focused on how students used their devices and likes/dislikes related to the equipment. Interviews were recorded on paper or audiotape for qualitative analysis, using constant comparison to detect emergent themes (Glaser, 1978). First, the interview transcripts were triangulated with written notes made regarding the responses to the questions. The results were then reviewed to determine specific themes among comments. Utilizing the established themes as categories, the notes were reviewed a third time and coded according to the categories. Videotaped observations made twice a week of classes in the laboratory classroom were analyzed for evidence of mobile computing use. To answer the second question, student and teacher interview data related to motivation to learn were also analyzed using the constant comparison method. Although students were more implicitly asked about this aspect of the research through questions of likes and dislikes, teacher interview questions were more specific, and included questions such as: Do you think your students' attitudes toward school, motivation to learn, and/or self-efficacy was improved by their use of the mobile computing devices? How? Can you give examples for high, average, and lower achieving students? Student and teacher data were analyzed separately at first, and the results were compared to identify commonalities and differences in response patterns.

Data for the third research question consisted of student work samples collected using PAAM from GoKnow. PAAM transfers all student work to an offsite server whenever individual mobile computing devices are backed up locally. Work was obtained from four students selected in each class as high, medium, and low achieving, and in all but one fourth grade class, for selected special needs students. Work samples were analyzed for conceptual understanding, based on a framework developed by Newmann (Newmann \& Wehlage, 1995; Newmann, Bryk \& Nagaoka, 2001), which focuses on evidence of students' use of analytic skills, their depth of conceptual understanding, and their ability to communicate that understanding. (see Table 1). The framework provides rubrics for assessing student work, assigning numeric scores for each of three criteria (a score of 1 being the lowest, 4 the highest, with total scores ranging from 3 to 12). Scores were computed for all selected student work samples, and averaged and compared within and across classes by ability groupings.

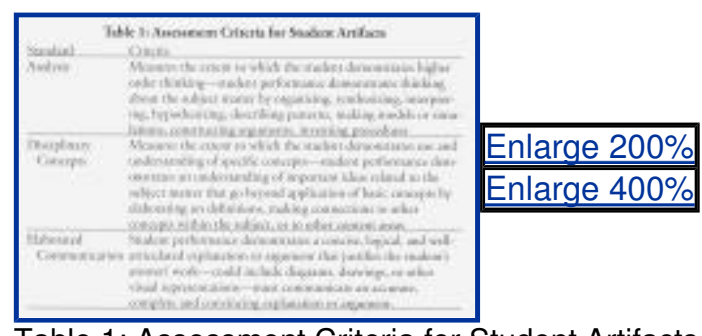

Table 1: Assessment Criteria for Student Artifacts

Student artifact scores were triangulated with teacher and student interview data, in order to determine whether mobile device usage can enhance student learning processes. Teacher and student data were analyzed separately and then compared to each other as well as the student artifact scores. 


\section{RESULTS}

Results from this preliminary study of the uses and effects of mobile computing devices are outlined in the sections which follow. They are organized by the research questions.

How do students use mobile computing devices?

All teachers in the study introduced their students to mobile computing, required the use of them for some explicit assignments (see Table 2), and encouraged students to use them as needed both inside and outside of class. The teacher at the middle school started out by requiring students to use the devices as much as possible, but a number of equipment issues forced her to make their use an option for the last three months of the school year. Despite the technical issues, most students continued to use their devices. Equipment failure was less of a problem in the laboratory classroom where technical support was constantly at hand,

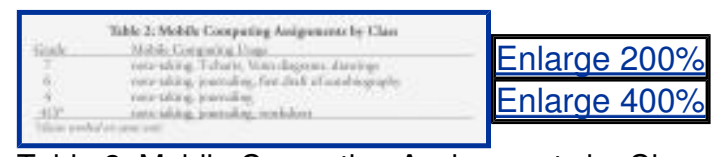

Table 2: Mobile Computing Assignments by Class

Table 2 shows the types of assignments teachers asked students to complete using mobile computing devices. All teachers required their students to use them for taking notes, and teachers in the laboratory classroom also gave journaling and other writing assignments. Interestingly, only the seventh grade science teacher-the teacher with the oldest students-required her students to use drawing programs. Nonetheless, usage data (see Table 3) reveals that all students involved in the study used drawing programs and that youngest students used them the most. Students in all classes also made considerable use of personal information management (PIM) applications such as the Date Book, Address Book, and To Do List, as well as the calculator, although such usage was considerably lower and is not shown in Table 3. Indeed, the most striking characteristic of the usage data is its variability, both between classes and between individuals, which suggests the ways in which mobile computing was appropriated by individual students and student cultures to personalize learning. Notice for example the significant differences between the two fourth grades and between fourth and third grade students given the exact same assignments.

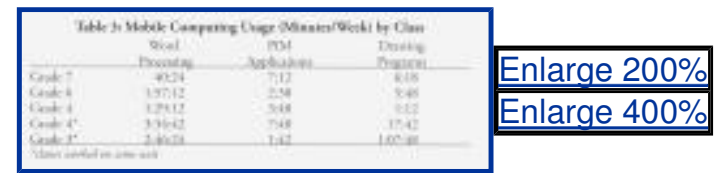

Table 3: Mobile Computing Usage (Minutes/Week) by Class

Table 3 also does not show the great variability between individual students within classes. In the sixth grade class, for example, one student used word processing for six hours a week, while students only used it for about one hour on average. A female student in this class spent two hours a week with word processing and another hour using the draw programs, while many students never used the draw programs. Two girls played with the PicChat functions; a few students used the calculator quite a bit while some students never used it; one student spent a good bit of time with Earth and Sun although most students barely looked at this application. Table 4 reflects this range of usage across classes, suggesting that at least some students in this study appropriated mobile computing devices for personal use (Roschelle \& Pea, 2002).

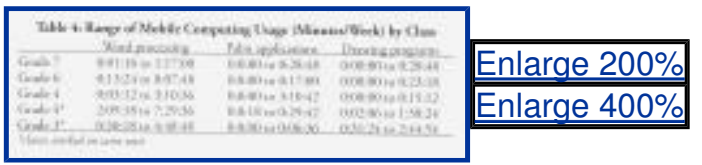

Table 4: Range of Mobile Computing Usage (Minutes/Week) by Class

The usage data findings are supported by the student interviews. More than $75 \%$ of the students interviewed reported using their mobile devices outside of the classrooms in which they were explicitly assigned-in other classes, at home, on the bus, and in after-school programs. The portability seemed a particularly important factor in their use. For example, when asked to compare mobile computing devices to desktop computers, one student told us "It's kind of like the same thing but it's smaller and easier to do. The computer you can't take with you wherever you go but the [mobile computing device] you can just close it up and take it wherever you go."

Students in the middle school classes reported using their mobile computing devices mostly for taking notes, while elementary students reported using them for a variety of writing activities, noting that they preferred using the devices to writing by hand. Many students also reported that they found them to be most useful for various types of 
organizational activities (scheduling, creating to-do lists, and outlining ideas). Students also reported enjoying the use of drawing programs and games.

Teachers also reported that students used the mobile computing devices in their classroom, at home, and on the bus to and from the laboratory classroom for writing assignments, journaling, note-taking, drawing, concept maps, spelling lists, test review, and to do lists. The third and fourth grade teachers who collaborated also commented that the beaming feature (i.e. infrared file transfer from one device to another) was particularly effective for facilitating the peer editing process. Several teachers noted that when mobile computing homework was assigned, all students completed it on time, something that almost never happened with paper and pencil assignments. One suggested that this was as much because using the mobile computing device helped students organize their work as it was a result of increased motivation. Indeed, one seventh grader stated that when using his device "I don't lose homework like with papers." This possibility deserves further exploration.

Table 5 compares mobile computing usage across classes by gender. These data show a tendency for girls to use mobile computing devices more than boys. As by far the most frequent use of the devices was for word processing, it may be that this occurred because girls tend to write more than boys, but the result clearly deserves further investigation in light of common findings of the opposite with respect to desktop computers. The gender data also highlight the variability between classroom cultures. Note, for example, the usage among sixth graders; boys in this class used their devices more than girls, suggesting something different happening among at least a group of boys in this class.

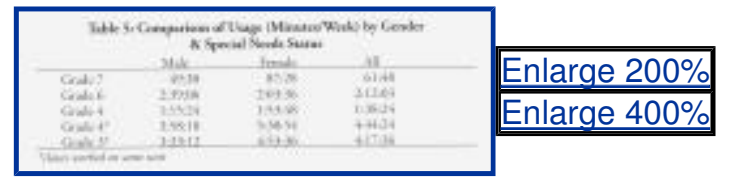

Table 5: Comparison of Usage (Minutes/Week) by Gender \& Special Needs Status

To summarize, our preliminary findings indicare that the use of mobile computing devices can be beneficial for learning inside and outside the confines of the classroom. This transition of use in and outside of school tends to be seamless, because students are using the same device with the same settings, files, etc., wherever they go. Therefore, learning that may have been taking place already outside of the classroom can be amplified by this technology as well, and should be subject to further study. The data also suggest unique cultures of use evolved within classes and groups within classes (e.g., by gender), indicating higher levels of personal appropriation. In addition, the results indicate that usage tends to be personalized as individual students adapt the use of mobile computing devices to their own needs. Finally, the findings indicate that, at least in this study, mobile computing devices were used most frequently for writing activities (potentially due to the fact that the device used had a built-in keyboard) and that in most cases such use was readily embraced by students. These findings clearly invite further investigation and should inform future research.

Does the use of mobile computing devices affect students' motivation to learn and engagement in learning?

Most teachers interviewed agreed that their students' motivation to learn and engagement in learning activities was improved by their use of mobile computing, which resulted in increased student productivity and improved quality of work. For example, the sixth grade teacher reported that

Taking the [mobile computing devices] home resulted in everyone's homework always being done, and shortened the time frame for getting work done. Having the [mobile computing devices] also improved the writing of all students.

One of the fourth grade teachers believed that mobile computing devices would be highly engaging in a regular classroom, and observed that "practice makes perfect" when it comes to the writing process, "the one benefit I've noticed is that they do write more with the [mobile computing devices]. And I believe that as much as it occurs with reading, the more you write, the better a writer you become."

This same fourth grade teacher, although noting his students' initial engagement with the devices, also reported that excitement about using them seemed to die off in favor of the more powerful desktop and laptop computers available to them. It should be noted, however, that this class's culminating projects included choices of creating Web pages and desktop videos, projects that are both highly motivating and which can only be done on regular computers. The findings here may be an indication that students were making choices with regards to which tools were most appropriate or worked best for particular tasks or projects. This finding is one that warrants further investigation, as making these kinds of choices is one of the pillars on which the success of ubiquitous computing environments for learning rests. 
Interviews with students confirm these findings. Students said that they preferred using the mobile devices over writing by hand and that using them for writing assignments made the work "easier" and "more fun." One student noted, for example, that "my writing is poor and the [mobile computing device] makes it easier to read my writing." Students also noted that using mobile computing devices helped them keep their work organized. One student commented, "I don't lose homework like with papers."

Several students commented that they liked being able to share their writing with their friends using the device's beaming capabilities. Although more than a few students noted difficulties and frustrations with the devices, including programs freezing, problems with recharging and hot syncing, losing work, and in a few cases difficulties with the keyboard and the stylus, the majority of these students believed the benefits outweighed these difficulties. Students interviewed particularly liked the fact that they could take their devices everywhere. Mobile computing devices thus enable working on school assignments or exploring personal interests whenever and wherever students are inclined to do so, and seem to be a critical factor in supporting motivation and engagement in learning. It certainly deserves further study.

Videotaped observations of students in the laboratory classroom also provide confirmatory evidence of student engagement in learning when working with mobile computing. Of particular note is their use of the devices for recording data from a variety of experiments undertaken in several of the participating classes. The videotapes show students as engaged in recording data as they are in doing the experiments. Perhaps this is because, as one student noted, the use of mobile computing makes such activities seem more like what "real scientists do." It may also be that the ability to easily carry the devices anywhere, their capacity to collect and store a variety of information, and directly input this into desktop computer applications alleviates much of the drudgery of working with data and supports inquiry learning. As one student commented, "It's really fun. It's nicer than doing it on paper."

In summary, findings show that the use of mobile computing devices can increase student motivation and engagement in learning, especially their motivation to complete written assignments. Findings also indicate that students' motivation to learn and engagement in learning with mobile computing devices may be decreased by equipment problems and/or access to competing technologies. The latter may not actually be a disadvantage, as the availability of a variety of devices for learning will force students to make informed choices about appropriate technology use. Research is needed to further explore these findings.

Does students' use of mobile computing devices support learning processes?

Teacher interviews indicate that mobile computing devices have the potential to enhance learning processes, especially with respect to writing. One teacher commented that the use of mobile devices resulted in noticeable improvements in both the peer editing process and the quality of student writing. She stated,

The biggest change has been in their weekly journals. We have been journaling all year and they have always written them but in using the [mobile computing devices], peer editing takes on so much more meaning when they can beam to someone rather than trading papers. With the [mobile computing devices] they are editing their own writing more and it keeps getting better.

Many teachers also made comments about improvements in spelling and mechanics as a result of more time spent on writing and editing. Perhaps most important, most teachers also noted improvements in the written work of special needs students. One teacher stated, "Having the [mobile computing devices] improved the writing of all students but special education students in particular." Another noted that "the special education students were empowered to write."

Indeed, many of the teachers interviewed commented on ways in which the use of the mobile computing devices seemed to lessen the gap in academic achievement between regular and special needs students. This observation is supported by work samples obtained from the 3-4 targeted students per class in the laboratory classroom, and analyzed for evidence of conceptual understanding (on a scale of 3-12, with 12 being the highest). Average results of these analyses across classes are given in Table 6 . The results indicated that for the classes in the laboratory classroom special needs students tended to perform on a level similar to medium ability students, while the seventh grade special needs students in science fell somewhere between low and medium ability peers. Analysis of lessons indicates that the overall lower ratings for the seventh grade science students may reflect assignments that did not elicit higher-order thinking, and this may have affected the performance of the special needs students.

Nonetheless, the results provide some evidence for positive effects of mobile computing, particularly when supporting the learning of special needs students.

Interviews with students support findings that the use of mobile computing devices may enhance student learning processes. For example, fifteen of the eighteen seventh grade science students interviewed stated that they believed their use of mobile computing helped them in their school work. These students particularly noted their 
helpful use for taking notes, test review, and doing calculations, while keeping their work on the devices helped them stay more organized.

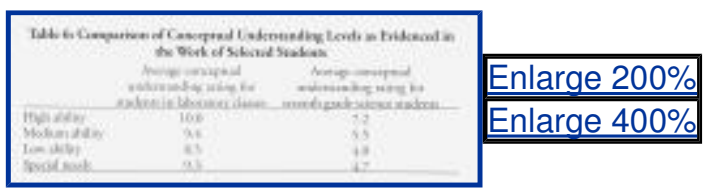

Table 6: Comparison of Conceptual Understanding Levels as Evidenced in the Work of Selected Students

As previously noted, students in the laboratory classes reported that they preferred using mobile computers over writing things by hand and that using mobile computing for writing assignments made the work "easier" and "more fun." This was even the case for the fourth grade students who preferred the desktops and laptops for higher-end applications such as multimedia presentations, Web design, and video editing. The majority of students in these classes also stated that they thought their written work in particular improved as a result of their use of the devices. For example, one student stated, "My writing is poor and the [mobile computing devices] makes it easier to read my writing." Indeed, all students interviewed seemed to view mobile computing devices as a tool that could help them with their school work. This aspect of the use of such devices surely deserves further investigation.

In summary, findings from this preliminary study provide some indication that the use of mobile computing devices can enhance student learning processes, especially when it comes to writing-an activity that the devices used in this study are well suited for, given the availability of a built-in, full-size keyboard. Perhaps more important, the results suggest a lessening of the gap in conceptual understanding levels between regular and special needs students using mobile computing devices, but only when assignments elicit it. Interviews with teachers suggest that the use of mobile computing resulted in greater productivity and improved writing skills among their students. Findings also suggest that mobile computing devices may provide increased support for schoolwork (especially the writing process) and levels of conceptual understanding. These findings surely deserve further investigation and moreover should inform future research.

\section{CONCLUSIONS}

This preliminary investigation of the use of mobile computing devices shows that elementary and middle school students use them in a variety of ways, principal among; these writing, both in and outside of class. The findings suggest both the personalization of learning supported by such devices and their potential usefulness in amplifying learning that may already be happening beyond the classroom. They also suggest that students easily adapt the use of mobile computing devices to their own needs and hint at the influence of classroom cultures on this appropriation. The findings hint at collaborative uses of such devices as well, especially during the editing process. The results of this study further indicate that use of mobile computing devices may increase student motivation to learn and increase their engagement in learning activities, which in turn, could lead to an increase in time spent on learning activities and higher quality work.

However, this study has some limitations that need to be considered when interpreting the findings above. For one, as with any technology that is introduced in a learning environment, there is always a novelty effect. Students tend to be more motivated to use a new piece of technology for learning because it is new. Second, the use of the mobile technology was only studied for a relatively short period of time. Further study is needed to determine whether motivation to use and levels of use change over longer periods of time, say three to five years. Third, because some of the data collected is self-reported, there is always the chance of a Hawthorne effect, i.e. participants report what they think the researchers expect to hear or see, not what is actually happening.

On a less positive note, the results indicate that equipment problems can constrain the use of mobile computing devices. These findings suggest that special attention needs to be paid to classroom logistics, equipment maintenance, technical support, and perhaps professional development for teachers using mobile computing options. Future studies should investigate ways of better supporting teachers and classes using such devices (see also Vahey \& Crawford, 2002; van 't Hooft, Diaz, \& Swan, 2004).

Finally, the observation that students have the opportunity to choose a technology tool for a specific task or activity needs closer investigation, as it may provide a clearer insight into how ubiquitous computing environments can be optimized for learning. Technology in itself won't make the difference; it's what students do with it that does.

\section{[Footnote]}

1 The devices under investigation in this research project are AlphaSmart's Danas. A Dana is a portable computer that can be described as a lightweight, oversized handheld computer with a touch screen, infrared port, and built-in keyboard. The operating system is Palm OS. The devices were donated by AlphaSmart as part of a research grant, and are referred to as "mobile computing devices" throughout the remainder of this article. 


\section{[Reference]}

References

Bannasch, S. (1999). The electronic curator: Using a handheld computer at the Exploratorium. Concord Consortium Newsletter. Retrieved August 10, 2003 from http://www.concord.org/library/1999fall/electronic-curator.html.

Cole, H., \& Stanton, D. (2003). Designing mobile technologies to support co-present collaboration. Personal and Ubiquitous Computing, 7, 365-371.

Danesh, A., Inkpen, K., Lau, R, Shu, K., \& Booth, K. (2001). Geney: Designing a collaborative activity for the Palm handheld computer. Proceedings of $\mathrm{CHI}$, Conference on Human Factors in Computing Systems. Seattle: WA. Glaser, B. (1978). Theoretical sensitivity: Advances in the methodology of grounded theory. Mill Valley, CA: Sociology Press.

Inkpen, K. (2001). Designing handheld technologies for kids. Proceedings of CHI, Conference on Human Factors in Computing Systems. Seattle: WA.

Mandryk, R. L., Inkpen, K. M., Bilezkjian, M., Klemmer, S. R., \& Landay, J. A. (2001). Supporting children's collaboration across handheld computers. Proceedings of $\mathrm{CHI}$, Conference on Human Factors in Computing Systems. Seattle: WA. Newmann, F. M., Bryk, A. S., \& Nagaoka, J. K. (2001). Authentic intellectual work and standardized tests: conflict or coexistence. Chicago, IL: Consortium on Chicago School Research.

Newmann, F. M., \& Wehlage, G. G. (1995). Successful school restructuring: A report to the public and educators. Madison, WI: Document Service, Wisconsin Center for Educational Research.

Roschelle, J. (2003). Unlocking the value of wireless mobile devices. Journal of Computer Assisted Learning 19, $260-272$. Roschelle, J., \& Pea, R. (2002) A walk on the WILD side: How wireless handhelds may change computer-supported collaborative learning. International Journal of Cognition and Technology, 1(1), 145-272.

Sharpies, M. (2000). The design of personal mobile technologies for lifelong learning. Computers and Education, 34, 177193.

Soloway, E., Norris, C., Blumenfeld, P., Fishman, B., Krajcik, J., \& Marx, R. (2001). Devices are ready-at-hand. Communications of the ACM, 44(6), 15-20.

Scaudt, C., \& Hsi, S. (1999). Synergy projects and pocket computers. Concord Consortium Newsletter. Retrieved August 10, 2003, from http://www.concord.org/library/1999spring/synergyproj.html.

Tinker, R. (1997). The whole world in their hands. Concord Consortium. Retrieved August 10, 2003, from http://www.concord.org/library/pdf/future.pdf.

Tinker, R., \& Krajcik, J. (Eds.). (2001). Portable Technologies: Science Learning in Context. New York: Kluwer Academic/Plenum Publishers.

U.S. Department of Education (2004). National Educational Technology Plan. Washington, DC: Author. Vahey, P., \& Crawford, V. (2002). Palm Education Pioneers Program: Final evaluation report. Menlo Park, CA: SRI International.

van 't Hooft, M., Diaz, S., \& Swan, K. (2004). Examining the potential of handheld computers: Findings from the Ohio PEP project. Journal of Educational Computing Research, 30(4), 295-312.

van 't Hooft, M., \& Swan, K. (2004). Special issue on ubiquitous computing: Introduction. Journal of Educational Computing Research, 30(4), 275-279.

Weiser, M. (1991). The computer for the 21st century. Scientific American, 265(3), 94-95, 98-102.

\section{[Author Affiliation]}

Karen Swan

Mark van 't Hooft

Annette Kratcoski

Kent State University

Darlene Unger

Virginia Commonwealth University

\section{[Author Affiliation]}

\section{Contributors}

Karen Swan is the RCET Professor in the Research Center for Educational Technology at Kent State University. She has published and presented both nationally and internationally in the specific areas of programming and problem solving, computer-assisted instruction, hypermedia design, technology and literacy, and asynchronous online learning. Her current research focuses on the latter as well as handheld technologies and teaching and learning in ubiquitous computing environments. (Address: Karen Swan, 201 Moulton Hall, Kent State University, Kent, OH 44242; kswan@kent.edu.) Mark van 't Hooft is a senior researcher and technology specialist in the Research Center for Educational Technology at Kent State University. His research focus is on the use of handhelds in K-12 and teacher education. Prior to his work at RCET, Mark taught middle school and high school social studies and language arts. He is also a co-founder and chair of the ISTE Special Interest Group for Handheld Computing (SIGHC). (Address: Mark van 't Hooft, 201 Moulton Hall, Kent State University, Kent, OH 44242; mvanthoo@kent.edu.)

Annette Kratcoski is a senior researcher in the Research Center for Educational Technology at Kent State University. She is currently one of the lead investigators on a research study examining the effect of various technologies on the authenticity and intellectual complexity of student work. She has also coordinated evaluations of various technology initiatives, including the evaluation of Ohio's Technology Literacy Challenge Fund and the external evaluation for various PT3 grants. (Address: Annette Kratcoski, 201 Moulton Hall, Kent State University, Kent, OH 44242; akractos@kent.edu.) Darlene Unger is a faculty member at Virginia Commonwealth University, where she works as a research associate with the Rehabilitation Research and Training Center on Workplace Supports. Dr. Unger's research activities have focused on 
employment of people with disabilities and the use of handheld technology to improve the educational and transitional outcomes of students with disabilities. She has previously coordinated federally funded grant projects that focused on the school-to-work transition of youth with disabilities. (Address: Darlene Unger, Rehabilitation Research and Training Center on Workplace Supports, Virginia Commonwealth University, 1314 W. Main Street, Richmond, VA 23284-2011; ddunger@vcu.edu.)

\section{References}

References (20)

Cited by (28)

\section{Indexing (document details)}

Subjects:

Locations:

Author(s):

Author Affiliation:

\section{Document types:}

Document features:

Publication title:

Source type:

ISSN:
Teaching methods, Elementary school students, Motivation, Information technology, Teaching aids \& devices

Ohio

Karen Swan, Mark van 't Hooft, Annette Kratcoski, Darlene Unger

Karen Swan

Mark van 't Hooft

Annette Kratcoski

Kent State University

Darlene Unger

Virginia Commonwealth University

Contributors

Karen Swan is the RCET Professor in the Research Center for Educational Technology at Kent State University. She has published and presented both nationally and internationally in the specific areas of programming and problem solving, computer-assisted instruction, hypermedia design, technology and literacy, and asynchronous online learning. Her current research focuses on the latter as well as handheld technologies and teaching and learning in ubiquitous computing environments. (Address: Karen Swan, 201 Moulton Hall, Kent State University, Kent, $\mathrm{OH}$ 44242; kswan@kent.edu.)

Mark van 't Hooft is a senior researcher and technology specialist in the Research Center for Educational Technology at Kent State University. His research focus is on the use of handhelds in $\mathrm{K}-12$ and teacher education. Prior to his work at RCET, Mark taught middle school and high school social studies and language arts. He is also a co-founder and chair of the ISTE Special Interest Group for Handheld Computing (SIGHC). (Address: Mark van 't Hooft, 201 Moulton Hall, Kent State University, Kent, OH 44242; mvanthoo@kent.edu.)

Annette Kratcoski is a senior researcher in the Research Center for Educational Technology at Kent State University. She is currently one of the lead investigators on a research study examining the effect of various technologies on the authenticity and intellectual complexity of student work. She has also coordinated evaluations of various technology initiatives, including the evaluation of Ohio's Technology Literacy Challenge Fund and the external evaluation for various PT3 grants. (Address: Annette Kratcoski, 201 Moulton Hall, Kent State University, Kent, $\mathrm{OH}$ 44242; akractos@kent.edu.)

Darlene Unger is a faculty member at Virginia Commonwealth University, where she works as a research associate with the Rehabilitation Research and Training Center on Workplace Supports. Dr. Unger's research activities have focused on employment of people with disabilities and the use of handheld technology to improve the educational and transitional outcomes of students with disabilities. She has previously coordinated federally funded grant projects that focused on the school-to-work transition of youth with disabilities. (Address: Darlene Unger, Rehabilitation Research and Training Center on Workplace Supports, Virginia Commonwealth University, 1314 W. Main Street, Richmond, VA 23284-2011; ddunger@vcu.edu.)

Feature

References, Tables

Journal of Research on Technology in Education. Eugene: Fall 2005. Vol. 38, Iss. 1; pg. 99, 14 pgs

Periodical

15391523

ProQuest document ID: 905949841 
Text Word Count

Document URL:
5934

http://ts.isil.westga.edu/login?url=http://proquest.umi.com/ pqdlink? $\mathrm{did}=905949841 \& \mathrm{Fmt}=4 \&$ clientld $=30336 \& \mathrm{RQT}=309 \& \mathrm{VName}=P Q D$

Copyright @ 2011 ProQuest LLC. All rights reserved.

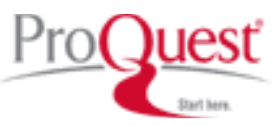

